\title{
DEL LIENZO EN BLANCO AL PLAYBACK. MODELOS DE IMPROVISACIÓN LIBRE EN EL ARTE TECNOLÓGICO
}

\author{
Jaime Munárriz Ortiz \\ Universidad Complutense de Madrid \\ munarriz@art.ucm.es
}

\section{RESUMEN}

El live coding, live cinema o distintos tipos de performance audiovisual desarrollados con sistemas digitales se proponen como nuevas formas de creación basada en la improvisación libre. Esto implica el uso de herramientas digitales que permiten la práctica performativa ante la audiencia, software y sistemas de programación audiovisual que se han desarrollado recientemente para este propósito. Estas prácticas tratan de mostrar al público los mecanismos de construcción de contenidos que subyacen en los sistemas digitales, sin ocultar los procesos técnicos que suelen quedar reservados al taller del creador. El deseo de lanzarse a una creación desde cero mostrando a la audiencia todo el desarrollo del proceso se contradice con la necesidad de establecer mecanismos seguros que resuelvan las dificultades tecnológicas. Utilizaremos la imagen del lienzo en blanco, con su problemática sobre el bloqueo creativo, para analizar estas prácticas artísticas en los nuevos medios y evidenciar las contradicciones y ficciones que implica este modelo de creación basado en la improvisación libre desde la nada a vista del público.

PAlabras Clave: improvisación libre, live coding, performance audiovisual, arte tecnológico, playback.

FROM BLANK CANVAS TO PLAYBACK.

FREE IMPROVISATION MODELS IN TECHNOLOGICAL ART

\section{Abstract}

Live Coding, Live Cinema or different approaches to digital audiovisual performance are proposed as new forms of creation based on free improvisation. This implies the use of digital tools that allow the performative practice in front of the audience, specialized software and audiovisual programming systems that have been developed recently for this purpose. These practices try to show the public the content building mechanisms subjacent to digital systems. Desire to get into a creative process from scratch showing the whole process contradicts the need of establishing safe mechanisms to solve the technical constrictions. We will use the concept of the blank canvas, with its problematic issues about the creative blockade, to analyse these artistic practices in the new media and to show the contradictions and fictions that lie behind this model of creation based on free improvisation from scratch in public view.

KEYwORDs: free Improvisation, live coding, audiovisual performance, technological art, playback. 


\section{INTRODUCCIÓN}

En la segunda década del siglo xxi se ha consolidado una práctica performativa que comienza a principios de siglo: el live coding. Consiste en la realización de actuaciones en directo con herramientas de programación y partiendo de una página en blanco (figura 1). El artista comienza, desde cero, a escribir el código a vista del público. El contenido sonoro o visual se va generando en tiempo real. No existe una versión terminada de la pieza, sino que el proceso mismo es la obra ofrecida a los asistentes. Este tipo de acción parece arriesgada, ya que cualquier error en el código, algo muy frecuente en la programación, supone un fallo ante la audiencia que puede ser de cualquier magnitud, desde un pequeño problema hasta una detención total del sistema. Se trata de presentarse ante el público sin red en una acción casi predestinada al fallo. Este comportamiento arriesgado constituye un valor esencial en esta escena. Presentar la creación con herramientas digitales como algo frágil y propenso al error es parte del valor de estas propuestas. Se acercan de este modo a las exploraciones del glitch y la transcodificación, indagaciones sobre la naturaleza de la información digital, y a la estética del error. Exponen los procesos internos de los sistemas digitales, normalmente ocultos bajo capas de interfaz gráfico que esconden los procesos y cálculos internos.

La escena de live coding disfruta de una salud envidiable ${ }^{1}$, repleta de energía y ganas de crear, si observamos las distintas actividades públicas que se realizan en la escena internacional: talleres, algoRaves, seminarios y conciertos como los realizados en lugares artísticos (medialabs, conservatorios, centros de arte independientes $^{2}$ ) de la práctica totalidad del planeta (Praga, Londres, Nueva York, Amsterdam, Madrid, Fráncfort, México, París, Madison, Bath, Buenos Aires, Richmond, Hamilton, Estambul, Moscú $\left.{ }^{3} ..\right)$. Suelen desarrollarse en forma de talleres y cursos, con eventos performativos que muestran los resultados de los encuentros. Nuevas herramientas de programación adecuadas a este tipo de acción han facilitado su desarrollo, además de las comunidades en la red.

Analizaremos la problemática de proceso y concepto que presentan estas prácticas que ofrecen una creación desde cero ante el público como núcleo central de su propuesta, estableciendo paralelismos con otras prácticas artísticas que se han enfrentado a problemas similares, como la improvisación libre, el live cinema o la música electrónica realizada con software específicamente diseñado para la actuación en directo como Ableton Live. Trataremos de establecer las dificultades operativas y conceptuales que subyacen a estas propuestas y su intento de llevar el proceso creativo ante la audiencia, cuestionando ciertos mitos que las sustentan y justifican,

${ }^{1}$ K. Burland, y A. McLean, «Understanding live coding events», International Journal of Performance Arts and Digital Media, 12, vol. 2 (2016): 139-151.

2 Peter Kirn, «Live coding group toplap celebrates days of live streaming, events», Create DigitalMedia, http://cdm.link/2019/02/live-coding-group-toplap-celebrates-days-of-live-streamingevents/ (consultada el 15 de febrero de 2019).

${ }^{3}$ Algorave, website, https://algorave.com/ (consultada el 11 de noviembre de 2019). 


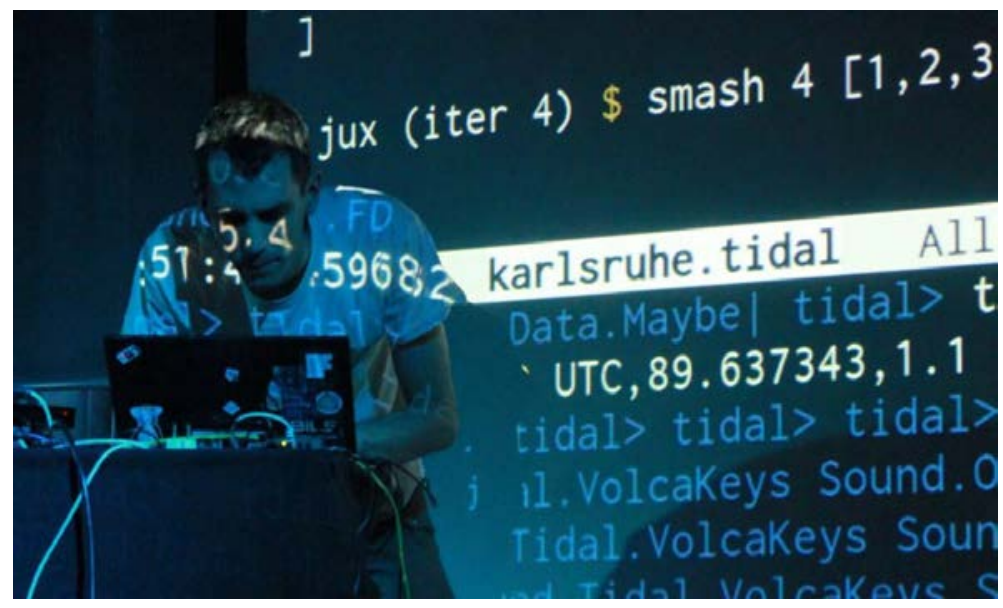

Figura 1. Live coding, Alex McLean (Yaxu).

a menudo en contra de la calidad del contenido producido, o en contradicción con el concepto que las fundamenta.

\section{IMPROVISACIÓN LIBRE}

La presentación ante el público sin un contenido definido previamente es una práctica que se desarrolla desde hace tiempo en el contexto de la improvisación libre, conocida también como improv ${ }^{4}$. Con origen en el free jazz de los años 60 y las prácticas performativas de Fluxus, se ha afianzado en el siglo xxi como una corriente internacional consolidada dentro de la electroacústica, la música experimental y la electrónica ${ }^{5}$. Se trata de una escena muy activa y con un perfil ideológico bien definido y bastante radicalizado que la defiende como estrategia de resistencia. La improvisación se presenta como una forma de liberación política, un último reducto ante las estructuras de poder: «A form of resistance to the established order that generates a concrete alternative» ${ }^{6}$.

${ }^{4}$ Colin Green, «The free jazz collective», Reviews of Free Jazz and Improvised Music, 5 de junio de 2017, http://www.freejazzblog.org/2017/06/into-maelstrom-music-improvisation-and.html (consultada el 11 de noviembre de 2019).

5 Derek Bailey, «Free improvisation», Cortical Foundation, 30 de mayo de 2000, (archivado el 12 de febrero de 2012), https://web.archive.org/web/20080605044808/http://www.cortical. org/dbfree.html (consultada el 11 de noviembre de 2019).

${ }^{6}$ Mathieu Saladin, «Points of Resistance and Criticism in Free Improvisation», en Noise \& Capitalism. (Guipuzcoa: Arteleku, 2015), 134. 
Live coding e improv comparten ciertos rasgos conceptuales y estéticos: los artistas se presentan ante el público sin una obra previamente construida, partiendo de cero, y elaboran su trabajo ante la audiencia, exponiendo los fallos y problemas que se producen en su desarrollo. El proceso se constituye en contenido. La improvisación libre plantea cuestiones de comunicación entre distintos músicos, así como del intérprete con su instrumento. El Live coding trabaja estos mismos conceptos $^{7}$, con la gran diferencia de que el instrumento es siempre un sistema de programación, algo duro, frío, insensible y muy diferente a un instrumento musical analógico, objeto con el que el músico establece una relación simbiótica muy física. Ambas formas de improvisación plantean un trabajo sin partitura previa, una obra construida desde la nada ante el público. Sin embargo, en ambos casos los artistas suelen partir de un guion previo no escrito, una estructura o un conjunto de condiciones que pueden ayudar a conformar la pieza final. Indicaciones que pueden referirse al ambiente o emoción de cada parte, la evolución de la obra, la intensidad y su desarrollo temporal, entradas y salidas de instrumentación o cualquier otra forma de organización de los recursos expresivos de que disponen los participantes. Las notas, frases o patrones que se construyen finalmente no están preestablecidos y no existe una partitura previa. Sin embargo, hablar de una improvisación total no es real, ya que se trabaja con conceptos y acuerdos comunes que permiten la materialización de una idea bastante bien formada en la mente de los participantes con anterioridad a su ejecución.

El improvisador libre lucha contra estos conocimientos previos, estos condicionantes tratando de «desaprender» $\mathrm{y}$ «reaprender» habilidades de escucha e interpretación liberadas de tradición y contexto. Una actividad que implica placeres, riesgos y una profunda implicación personal como individuo:

Expression, creativity, intuition, free play, raw material, risk [...] musical improvisation as an end in itself, though depend on all these life-affirming qualities, is also an experimental public struggle with the limits of the self, profoundly fulfilling and yet painful...8.

Hay que hacer hincapié en que el uso del término libre (free) carga de connotaciones ideológicas a todo el movimiento, que a menudo genera importantes polémicas en cuanto al entendimiento de lo que puede suponer esa libertad, como sabemos, un concepto complicado y elusivo.

7 Thor Magnusson y Alex McLean, «Performing with Patterns of Time» (Oxford: Oxford University Press, 2018).

${ }_{8}$ David Toop, Into the Maelstrom: Music, Improvisation and the Dream of Freedom: Before 1970 (Londres: Bloomsbury Publishing, 2016), p13. 


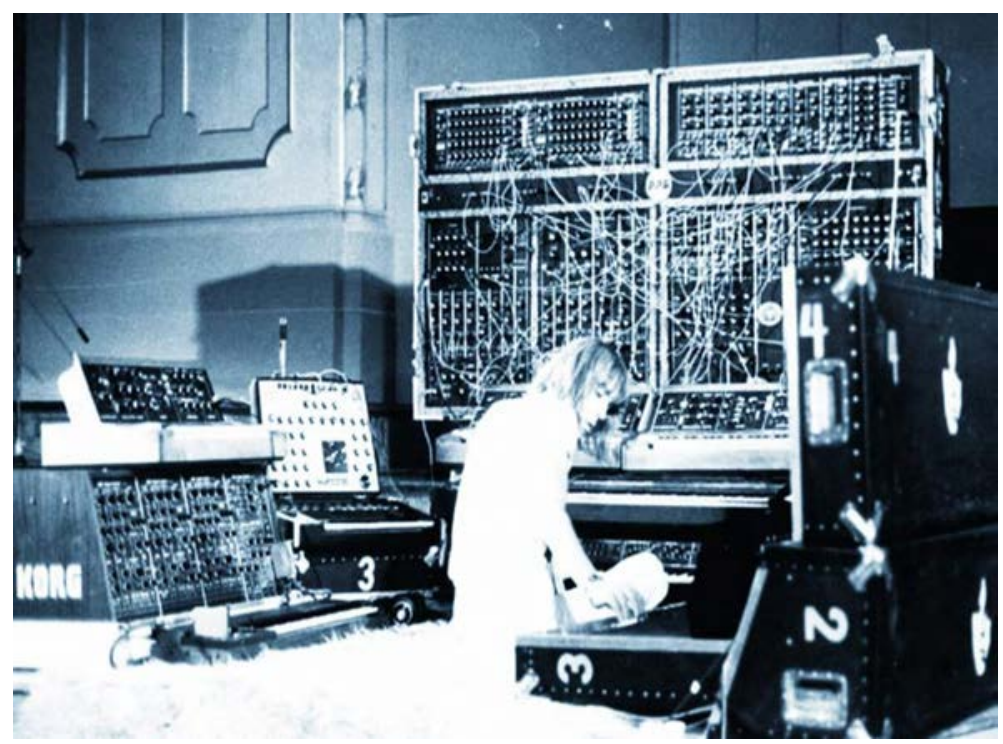

Figura 2. Klaus Schulze, Sintetizadores modulares.

\section{SECUENCIADORES Y PRESETS}

En el mundo de la música electrónica, la evolución de la tecnología ha condicionado fuertemente las opciones de desarrollo en directo y el espacio para la improvisación. Los sintetizadores y secuenciadores modulares de los años 70 , utilizados en el rock sinfónico y rock progresivo, necesitaban un largo proceso de ajuste de cada botón, cable y potenciómetro para conseguir un nuevo sonido (patch). De este modo se preparaban para el inicio de una actuación, y durante ella el músico podía ir transformando ese sonido inicial y las secuencias asociadas en nuevas mutaciones, pero siempre progresivamente, poco a poco, deslizándose desde un ajuste global hacia derivaciones del mismo. Un cambio completo del sistema resultaba imposible, porque el tiempo necesario impedía realizarlo en el momento durante una actuación. Por este motivo, los sintetistas necesitaban la presencia en el escenario de múltiples sintetizadores y secuenciadores para poder utilizarlos en distintos momentos de la actuación, cada uno con un ajuste específico (figura 2).

La tecnología digital introduce los presets o preajustes, que permiten cambiar con la pulsación de un solo botón todos los parámetros de un dispositivo. Esto parecería aportar mayor libertad al directo; sin embargo, los presets se convierten en una estructura demasiado rígida, que obliga a repetir las partes y ajustes de las composiciones de forma rigurosa. Se pierde la capacidad de transformación progresiva de estructuras y secuencias, esa transformación continua que construyó un nuevo universo electrónico en las primeras grabaciones de Tangerine Dream y demás artistas pioneros en la música con sintetizadores analógicos. 
Los presets llevan a los músicos de los años 80 a un territorio muy próximo al playback. Los sistemas digitales, sincronizados por MIDI (Musical Instrument Digital Interface), lanzan patrones y secuencias pregrabados. Los músicos pueden tocar por encima, pero estructuras, sonidos e ideas están completamente preparados de antemano, y son disparados mediante secuenciadores que repiten exactamente lo preprogramado.

\section{LAPTOPS}

La incorporación de ordenadores al proceso de elaboración de material musical aporta una gran flexibilidad. La composición se traslada poco a poco a los nuevos programas secuenciadores en ordenadores personales. Resulta difícil trasladar estas composiciones a escena, con lo que los intérpretes empiezan a subirse al escenario con un ordenador cargado con las bases de todo el espectáculo. Los músicos se ven obligados a seguir a este material pregrabado que no permite ningún tipo de modificación. Estructuras, instrumentación, tempo: todos los parámetros están prefijados, y en escena los músicos sólo pueden seguir esas bases que se reproducen en playback. Cubase, ProTools, Logic, los grandes secuenciadores, presentan un papel incómodo en un escenario. Son herramientas de estudio, aptas para la grabación y composición, y nunca estuvieron diseñadas para la interpretación en directo; mucho menos, para la improvisación. Los músicos electrónicos de la última mitad del siglo xx necesitan un enorme arsenal de dispositivos en directo para poder reproducir todos los matices del material elaborado en el estudio. Pueden modificar lo que suena, disparado por el ordenador, pero básicamente se limitan a ajustar el sonido, niveles de mezcla, envíos y efectos, realizando una suerte de remezcla en directo de un material completamente preformado.

La aparición del software Ableton Live en $2001^{9}$ cambia completamente este escenario. Detectando los problemas asociados a trabajar con el software existente para la grabación de estudio en el contexto de un escenario, Ableton Live se concibe como una herramienta para la interpretación en directo. La posibilidad de lanzar diferentes clips en cada pista, así como de mezclar y modificar con efectos virtuales todo el material sonoro, convierte a Ableton Live en una alternativa a todo el despliegue de equipo necesario para una actuación en directo con electrónica. Un único ordenador con Live puede realizar todas las funciones para las que antes se necesitaba una máquina dedicada para cada tarea, además del cableado: secuenciador, sámpler, sintetizador, caja de ritmos, mesa de mezclas, efectos... La idea es tan adecuada, entendiendo perfectamente las necesidades del músico electrónico del momento, que en seguida se convierte en la herramienta universal para la actuación

9 Future Music, "A brief history of Ableton Live», Music Radar, 13 de enero de 2011, https://www.musicradar.com/tuition/tech/a-brief-history-of-ableton-live-357837 (consultada el 11 de noviembre de 2019). 
en directo. Nacido dentro de la escena de música electrónica, es adoptado como herramienta apta para todo tipo de música en directo: clásica contemporánea, pop, rock, experimental, etc. Trasladar todo el sistema al interior de un simple laptop presenta innumerables ventajas para el desplazamiento, pero también para la creación de sistemas abiertos que permiten un alto grado de juego e improvisación. Sin embargo, nos lleva a un nuevo problema: trabajar sólo con un teclado y un ratón resulta en un sistema absolutamente frío y alejado de las necesidades expresivas y gestuales de un intérprete en directo. Para tratar de resolver este problema artistas e industria se lanzan a imaginar y proponer nuevos controladores físicos, aparatos que ofrezcan formas de interfaz adecuadas para la interpretación en directo.

\section{LIVE CINEMA}

Las prácticas de video jockey y live cinema sufren un proceso parecido: el software permite organizar contenidos que podemos lanzar libremente en un contexto performativo. Las ideas se preparan de antemano, pero podemos reorganizarlas y jugar con ellas libremente creando nuevos contenidos, jugando con superposiciones y manipulación de efectos y filtros. Estos sistemas permiten por primera vez la generación en tiempo real de narrativas improvisadas, montajes que juegan con asociaciones y encuentros entre secuencias e imágenes. La improvisación llega a la imagen proyectada ${ }^{10}$.

Esta tendencia tiene un practicante pionero excepcional: Peter Greenaway, con su proyecto Tulse Luper Suitcases ${ }^{11}$. En 2005 comienza un espectáculo de proyecciones en directo en el que remezcla el material de su película Tulse Luper stories, con un sistema táctil y multipantalla ${ }^{12}$. La comunidad de videojockeys llevaba trabajando en espectáculos similares desde una década antes, pero la irrupción de este director de cine experimental de reconocido prestigio impulsó un cambio hacia esta nueva forma de narratividad no lineal, saliendo del puro juego visual base de los orígenes del medio en el contexto de salas de baile con música electrónica. El Live cinema busca una nueva forma de narración, una narración líquida, que rompe la estructura fija del montaje cinematográfico. El realizador puede saltar de un plano a otro, creando superposiciones y jugando con efectos visuales. Se crea una historia por conexiones asociativas y connotaciones, una narrativa que es única y diferente

10 Jaime Munárriz, «Live Cinema: redefiniendo la narración audiovisual». Arte y Políticas de Identidad, vol. 9 (2013): 149-161.

${ }^{11}$ Lupercyclopedia VJ Performance, Peter Greenaway official site, (Archivado el 29 de mayo de 2010). https://web.archive.org/web/20100529060345/http://www.petergreenaway.info/ (consultada el 11 de noviembre de 2019).

${ }^{12}$ Benjamin Noys, «Tulse Luper Database: Peter Greenaway, the New Media Object and the Art of Exhaustion", Image and Narrative. Online Magazine of the Visual Narrative, agosto de 2005, http://www.imageandnarrative.be/inarchive/tulseluper/noys.htm (consultada el 11 de noviembre de 2019). 
en cada evento. La mayor dificultad creativa en esa búsqueda de libertad reside en la necesidad de utilizar material pregrabado, planos y escenas almacenados en el sistema que contienen su propia linealidad interior.

\section{LIVE CODING}

Las prácticas de live coding han necesitado de nuevas herramientas de software para poder desarrollarse. Los lenguajes de programación tradicionales no son adecuados para la actuación en directo. El proceso tradicional conlleva la escritura de código con un proceso posterior de depuración y compilación que genera un código «ejecutable» que puede finalmente lanzarse. No podemos realizar modificaciones al código sin detener la ejecución del programa que estamos generando, y volver a compilarlo y lanzarlo. C++, Java, Basic son algunos de los lenguajes más conocidos que siguen este modelo. Están pensados para construir un programa, pero no para una interacción continua con el código mientras se ejecuta, con lo que no permiten el live coding.

La aparición de Max/MSP y el desarrollo posterior de PureData realizado por el mismo autor, Miller Puckette, pero dentro del modelo del software libre, introduce un paradigma diferente en la creación de sistemas digitales multimedia. Este software nace en el IRCAM (Institute for Research and Coordination in Acoustics / Music) de París, un centro dedicado a la electroacústica. Desde el primer momento se concibe como un sistema que está siempre en ejecución, de modo similar a como los grandes sintetizadores modulares funcionaban continuamente mientras se cambiaban los cables o se accionaban interruptores y potenciómetros. El software está siempre funcionando. Se pueden añadir objetos en cualquier momento y cambiar conexiones entre ellos, construyendo un sistema siempre activo. No es extraño que esta nueva filosofía en el software invitara a explorar las posibilidades de la construcción de sistemas en tiempo real. Los artistas vislumbran las posibilidades de este tipo de práctica, y se lanzan a explorarlas en performances experimentales.

Hay que destacar el carácter innovador de una de estas primeras experiencias, por parte de uno de los pioneros en este campo: Enrique Tomás (quien a día de hoy trabaja en el centro Ars Electrónica de Linz). En 2006 Medialab Madrid organiza Radar, un evento dentro del programa "Interactivos?» de live coding con PureData y Arduino, en el que participan David Cuartielles, Gunther Geiger, Jaime Munárriz y Daniel González ${ }^{13}$. Enrique Tomás parte en esta ocasión de una ventana en blanco en PureData sobre la que comienza a construir un patch que poco a poco da forma a la pieza. Pero no se limita a trabajar con software; al mismo tiempo, armado de un soldador, fija cuatro potenciómetros en un trozo de cartón, suelda cables y los conecta a un Arduino, realizando delante del público un interfaz físico rudimentario, con el que luego maneja las ondas sinusoidales que se producen en PureData.

${ }_{13}$ Medialab Madrid, abril de 2006, http://www.medialabmadrid.org/medialab/interactivos.php (consultada el 11 de noviembre de 2019). 


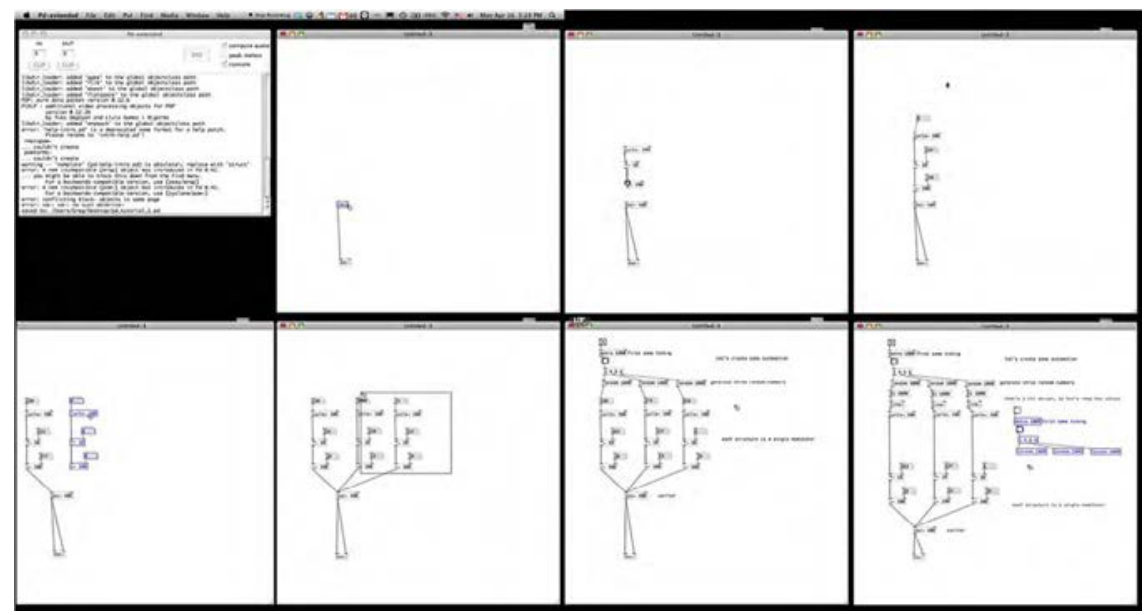

Figura 3. Greg Surges, PureData, Live Coding 1 - Multi-op FM.

Greg Surges, «Pure Data Live Coding 1 - Multi-op FM», 16 de abril de 2012,

https://www.youtube.com/watch?v=nLQMpUQ6It8 (consultada el 11 de noviembre de 2019).

Podemos contemplar un proceso similar en la figura 3, en la que poco a poco se va construyendo un sistema generativo que suena desde el principio y que va adquiriendo nuevas capacidades de control y expresión. En esta performance encontramos ideas pioneras para una nueva forma de generar contenidos, una nueva relación entre artista, instrumento y público. Una reflexión sobre nuestra relación con las herramientas digitales, el contenido y su relación con el sistema, y sobre el interfaz y sus aspectos expresivos.

\section{SUPERCOLLIDER Y SONICPI}

Otra rama del software, la de lenguajes funcionales como Smalltalk o Lisp, presenta un sistema de ejecución en tiempo real sin necesidad de compilación, que lo hace adecuado para la interpretación en directo. De este modelo nacen SuperCollider y SonicPi, sistemas que dan lugar a una nueva generación de artistas trabajando en live coding. Entre esta nueva generación podemos nombrar a Ron Kuivila, Slub, Alex McLean, Adrian Ward, Ge Wang, Sam Aaron, Joanne Armitage, Matthew Yee-King, Alexandra Cardenas, Nick Collins, Shelly Knotts y Benoît and the Mandelbrots ${ }^{14}$.

${ }^{14}$ Andrew R. Brown, «Code Jamming», Journal of Media and Culture, vol. 9, n. ${ }^{\circ} 6$ (diciembre 2006), http://journal.media-culture.org.au/0612/03-brown.php (consultada el 11 de noviembre de 2019). 
En estos sistemas tenemos un servidor, siempre activo, que se encarga de gestionar los eventos temporales con gran precisión, y un cliente sobre el que podemos interactuar, escribiendo órdenes que inician, modifican y detienen procesos. No existe una partitura o secuencia preestablecida. El artista introduce elementos y los modifica y detiene mediante comandos. El material se genera en tiempo real, y la estructura y la forma de la pieza se crean en directo, delante del público, que puede ver en una pantalla los comandos utilizados, así como los errores y otros problemas que puedan surgir. El artista que interviene en estas propuestas se sumerge en un proceso de integración con el sistema que requiere total concentración para lograr moverse en la complejidad. Un virtuosismo técnico que contrasta con la imagen abierta y cercana del artista, heredera del punk y su actitud desmitificadora: "Coding generally involves making sense out of huge, crazy structures, and it's impossible to get anywhere without zoning out into a state of focussed, creative flow $»^{15}$. Este proceso permite un flujo continuo de trabajo, un proceso de realimentación en el que la tecnología potencia las decisiones creativas y la integración del performer:

Live coders write code while a computer reads and processes it. This leads to an immediate feedback cycle, where the programmer is able to manipulate a running system, quickly trying out ideas and seeing, hearing or otherwise experiencing the results without any break of flow ${ }^{16}$.

El proceso de realimentación es fundamental en el sistema, convirtiéndose en centro del proceso creativo y expresivo. Existe una comunidad muy activa que organiza encuentros y eventos alrededor de live coding: Research Network. En España contamos con el papel realizado por Jesús Jara alrededor de Medialab Prado (figura 4) y su grupo "Live Code Mad», desde 2015. Otros miembros se encuentran en Alemania, México, Colombia, Inglaterra, Canadá. Desarrollan conciertos, talleres y eventos denominados Algorave, término muy afortunado que nace de la unión de las palabras rave y algoritmo. Esta nueva generación de artistas de live coding está siendo muy activa en los últimos años, como se puede comprobar en la plataforma toplap.org. Es de destacar la celebración del encuentro periódico International Conference on Live Coding, celebrado en diversos países, y la aparición de nuevas plataformas alrededor del lenguaje Javascript y sus capacidades para la creación visual, como Hydra (Olivia Jack), PraxisLive (Neil C. Smith) y Visual Fiha (Valentín Vago).

El live coding no sólo es una forma de improvisación libre. Aunque se parte de cero en cada actuación, lo importante de esta actitud está en evidenciar los procesos subyacentes en la generación de contenidos visuales y sonoros sin esconder-

15 Stephen Fortune, «What on earth is livecoding?», Dazed, 14 de mayo de 2013, http:// www.dazeddigital.com/artsandculture/article/16150/1/what-on-earth-is-livecoding (consultada el 11 de noviembre de 2019).

${ }^{16}$ Live Code Research Network. «What is live coding?», 3 de julio de 2015 (archivado el 26 de junio de 2012), http://www.livecodenetwork.org/what-is-live-coding/ (consultada el 11 de noviembre de 2019). 


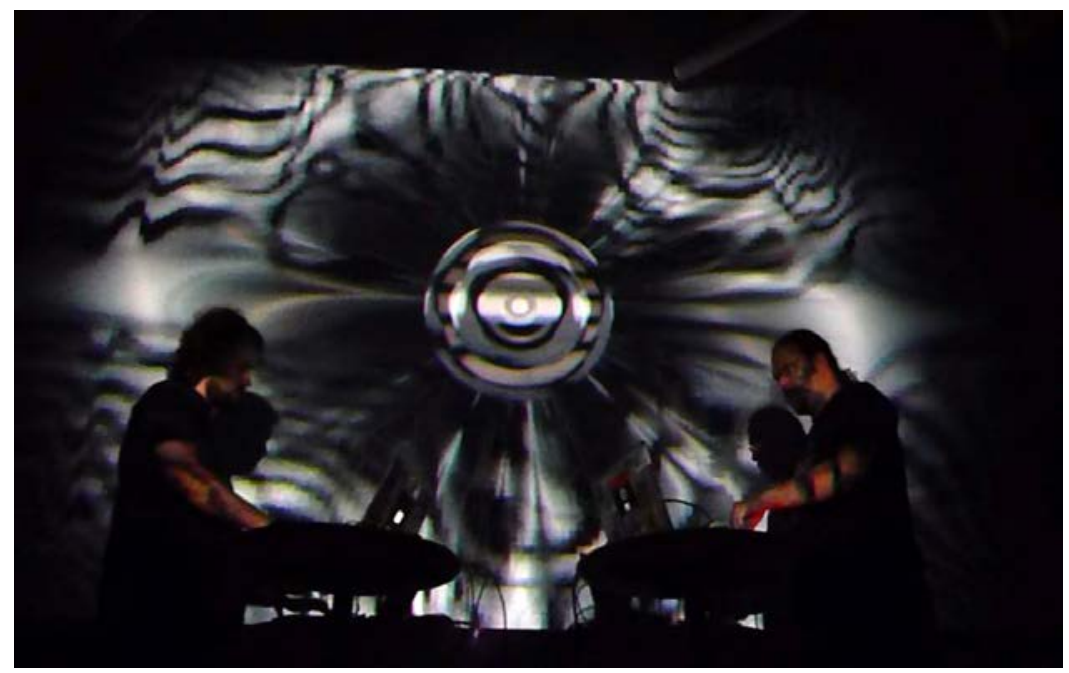

Figura 4. Ganzfeld, Medialab Prado.

los bajo interfaces ni contenidos llamativos: «Code should be seen as well as heard, underlying algorithms viewed as well as their visual outcome. Live Coding is not about tools. Algorithms are thoughts $\rangle^{17}$. Cansados de presenciar artistas escondidos tras la tapa de su ordenador, una nueva generación quiere ver qué está pasando en la pantalla. Los algoritmos se exponen ante el público, que puede comprender lo que está sucediendo en el sistema digital.

Live Coding allows us to perform with symbol rather than gesture. The ability to use abstraction in performance frees us from the need to gesture but can also allow us to tie complex behaviors to simple gestures. It brings performance much closer to language and therefore all of the advantages that using language gives us ${ }^{18}$.

La liberación del gesto en la interpretación es desde luego una cuestión controvertida, que se enfrenta a las nociones de genio y al romanticismo de la figura del intérprete. El empleo del código conduce sin embargo a un nuevo virtuosismo, un virtuosismo simbólico, que destaca las capacidades mentales frente a las corporales.

17 Toplab, «Manifesto Draft», 14 de noviembre de 2010, https://toplap.org/wiki/ManifestoDraft (consultada el 11 de noviembre de 2019).

${ }^{18}$ Andrea Mi, «Rave your algorithm. Interview with Renick Bell», Digicult, 26 de abril de 2018, http://digicult.it/articles/rave-your-algorithm-interview-with-renick-bell/ (consultada el 11 de noviembre de 2019). 
La promesa del live coding está en producir una obra ante la vista del público partiendo de cero, sin un trabajo de composición previo, escribiendo órdenes y comandos que ponen en marcha los procesos digitales. Del mismo modo, el software Ableton Live permite plantarse ante el público con un sistema completamente abierto, que permita una improvisación total, con la creación en directo de un material único y adecuado a cada ocasión. El live cinema participa de este mismo ideal: podemos presentarnos ante los espectadores con un sistema abierto, y crear piezas únicas que sólo pueden experimentarse una vez, en un contexto performativo único. La improv nos ofrece el mismo ideal: la improvisación libre como forma máxima de creación. Estas promesas de creación desde la nada, de partir de un lienzo en blanco ante el público para elaborar una pieza única no imaginada anteriormente, fruto de la maestría técnica y el genio creativo del autor, no son sin embargo desarrolladas habitualmente en la realidad. Se presentan como modelo utópico, pero son difíciles de lograr, tanto técnica como conceptualmente.

El lienzo en blanco es complicado. Cualquier creador conoce el problema del bloqueo ante una página, un lienzo o una pantalla en blanco ${ }^{19}$. Enfrentar el proceso de creación desde el lienzo en blanco al público es difícil, ya que no podemos estar seguros de poder ofrecer un resultado con una calidad que esté al nivel de las expectativas creadas. El artista necesita un cierto nivel de seguridad, una pequeña red que amortigüe la caída en caso de fallo total. De este modo tiende a utilizar una serie de recursos que le posibiliten llevar a cabo esta tarea de improvisación en público con el máximo grado de éxito posible. El creador lleva consigo una caja de trucos, habilidades y conocimientos que pueden conformar una experiencia adecuada, pero son recursos previamente preparados. La estructura, el desarrollo temporal de la pieza o la actuación es algo que se puede diseñar mentalmente y acordar en el caso de varios participantes. Un posible ejemplo de estructura acordada podría ser: «Empezar con una parte tranquila, luego pasar a..., subir de intensidad..., cambiar de ritmo..., coger otro instrumento..., lanzar un motivo..., realizar determinado gesto para un cambio...». La pieza cuenta con una planificación previa que le garantiza un desarrollo que evite la monotonía y el aburrimiento, y cierta seguridad a los intérpretes de alcanzar un resultado satisfactorio.

Cada performer o músico conoce su sistema o instrumento. Sabe qué operaciones puede realizar, qué frases funcionan. Ha probado y ensayado previamente los recursos disponibles, y cuenta con un repertorio de recursos con los que se asegura un éxito en su papel. Un instrumento físico permite un grado mayor de riesgo, y en la improv sí encontramos casos extremos en los que un intérprete puede enfrentarse a un instrumento desconocido o a una interacción extraña que nunca ha realizado. Por el contrario, en los ordenadores el código de programación es implaca-

19 Gustav Andersson, «Blank Canvas Paralysis», The modern nomad, 17 de febrero de 2012, https://www.themodernnomad.com/blank-canvas-paralysis/ (consultada el 11 de noviembre de 2019). 


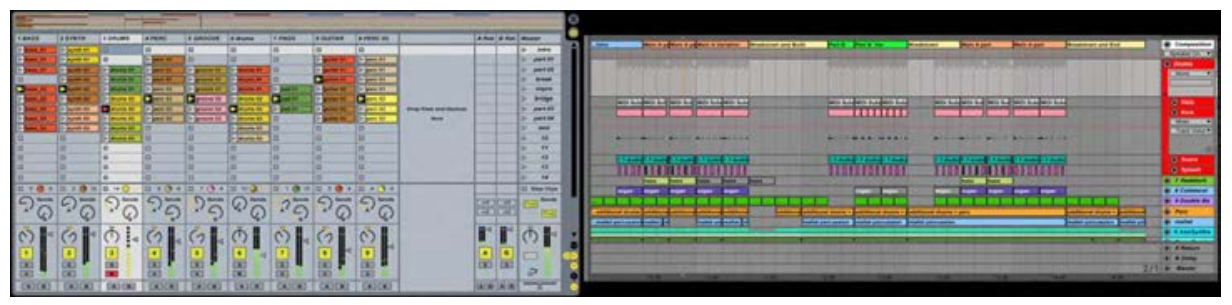

Figura 5. Ableton Live: de una sesión totalmente abierta al playback.

ble: sólo podemos introducir comandos válidos para el sistema. Todo lo demás es un error inútil, que no produce resultados, sólo un mensaje de aviso o en el peor de los casos el bloqueo del aparato. El artista del live coding tiene por tanto que llevar los deberes hechos, conocer perfectamente la sintaxis del lenguaje que utiliza y contar de antemano con una serie de elementos que puede ir introduciendo, sabiendo que van a funcionar. A menudo utiliza bloques de código ya preparados, que puede activar con una sola orden.

Todas las tendencias artísticas que hemos presentado tienen en común el mito del lienzo en blanco y la creación libre ante el público, una exposición performativa del proceso artístico. En la realidad, el artista se ve poco a poco arrastrado desde esa libertad total a una estructuración del material. Un set concebido para la improvisación se va poco a poco cerrando: hay combinaciones que funcionan, otras que no. Poco a poco el set de directo, si se va repitiendo en distintos eventos, empieza a fosilizarse, a congelarse. La pieza que inicialmente se concibe como algo completamente abierto acaba convirtiéndose en playback (figura 5).

Podemos observar esta tendencia incluso en el desarrollo de una de las herramientas de software utilizadas: Resolume, el programa más utilizado para lanzar vídeos en tiempo real pasa en una actualización de una retícula de celdas que podemos activar libremente a una estructura lineal. El funcionamiento es el mismo, podemos disponer de clips de vídeo y lanzarlos cuando queramos. Sin embargo, la lógica del interfaz introduce un factor de secuencia temporal (ver figura 6). Nuestro set completamente abierto se ve de este modo atrapado en una lógica de secuencia, perdiendo su modelo de libertad total.

La programación en directo sufre esta misma evolución: el creador acumula procesos mentales que sabe que funcionan, y los utiliza en una sucesión conocida. Incluso almacena ciertos bloques de código en archivos que puede lanzar con una sola orden. En este sentido no es muy distinto de un intérprete de música barroca, que conoce su instrumento y la partitura, y puede improvisar en algunos lugares y bajo determinados parámetros. Existe un cierto grado de improvisación y de libertad, pero siempre dentro de unos márgenes preestablecidos. La obra que nace en la mente del artista con el mito del lienzo en blanco y la libre improvisación acaba fijándose y estructurándose con el tiempo. Poco a poco se cierran opciones, se fijan algunas combinaciones, se consolida una estructura y se concretan los recursos a utilizar... hasta estar muy cerca del playback. Es un proceso común a diversas cate- 

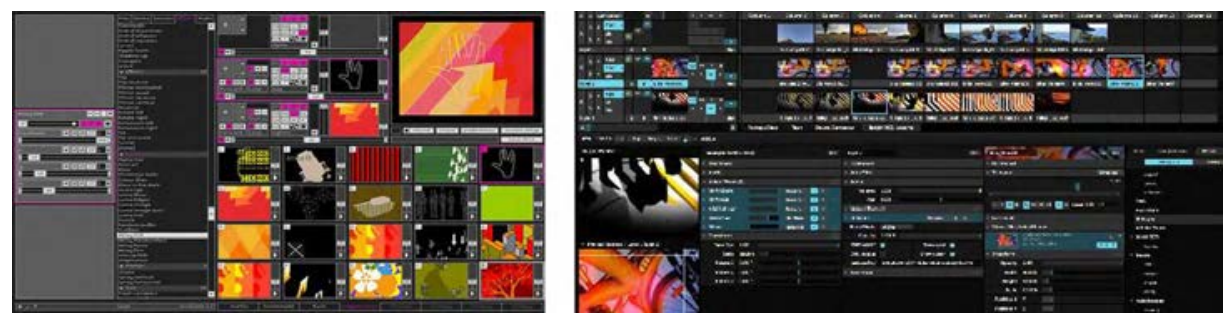

Figura 6. Resolume: de la rejilla abierta a la secuencia preestablecida.

gorías de artes que emplean la improvisación como estrategia. Un proceso que cierra opciones, concretando resultados funcionales y determinando un resultado óptimo y conocido.

En este equilibrio de libertades y compromisos tenemos que movernos en el contexto de la creación tecnológica contemporánea en directo. El artista diseña su sistema y establece límites y opciones. En la construcción del sistema se plantea la tensión entre parámetros predefinidos y márgenes de libertad e improvisación. La tecnología facilita algunas cosas, pero impone límites y barreras muy duros en otras. Jugar en este territorio se constituye como el acto artístico del nuevo paradigma tecnológico, un desafío en nuestra relación con el nuevo medio digital (figura 7): «The crucial questions are: What is being performed: music, notation, or the process of creation? And who is the performer and who is the audience? $\rangle^{20}$. El medio se convierte en contenido, el sistema en creador, el proceso y sus complejidades tecnológicas en el material que se presenta a la audiencia.

\section{CONCLUSIONES}

La improvisación libre se plantea como un ideal quimérico para el artista performativo en los medios visuales o sonoros. Cada tecnología ofrece opciones y límites y la tecnología digital ofrece unos límites duros que no permiten errores o indeterminaciones. Los marcos de acción establecidos en el sistema producen estructuras de comportamiento prefijadas que posibilitan el desarrollo de la pieza. Estos modelos nos alejan del lienzo en blanco para arrastrarnos peligrosamente al playback. Moverse con inteligencia en este territorio plagado de trampas conceptuales y escollos tecnológicos se plantea como el territorio más fértil pero más complicado para la creación tecnológica contemporánea.

${ }^{20}$ Martin Flašar (Brno.), «Listening with the Eyes: Remarks on Live Coding Performance», Media Archive Performance, n. ${ }^{\circ}$ 7, (2016), http://www.perfomap.de/map7/media-performance-on-gestures/listening-with-the-eyes-remarks-on-live-coding-performance (consultada el 11 de noviembre de 2019). 


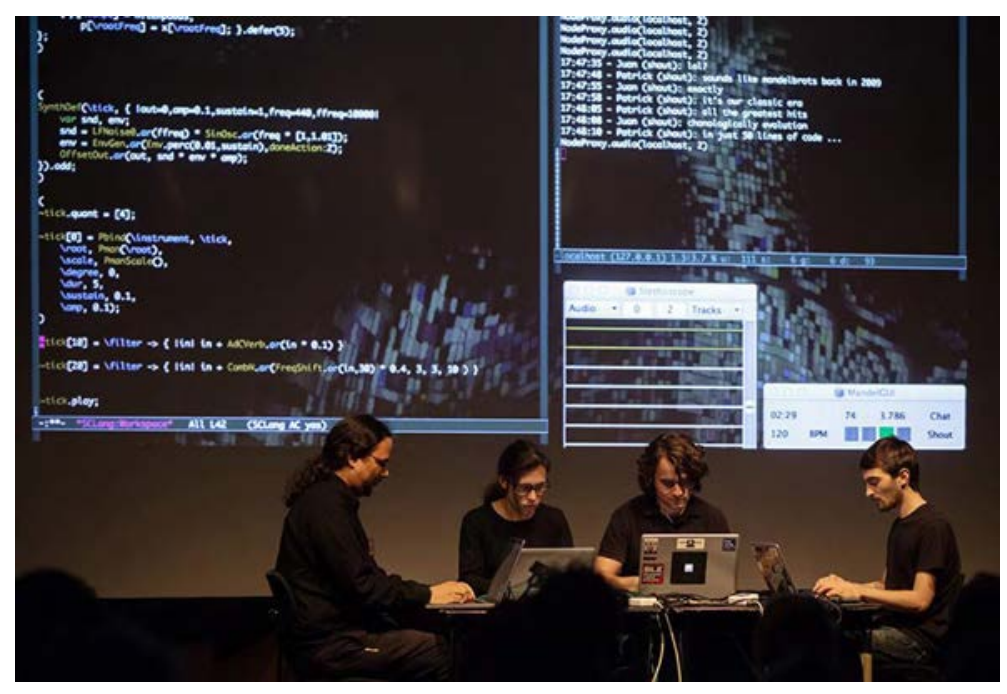

Figura 7. Benoit and the Mandelbrots.

Las tecnologías audiovisuales digitales han potenciado la aparición de distintas prácticas artísticas que han optado por convertir el proceso de creación y fabricación de una pieza en su propio contenido y en la cualidad estética y conceptual que las fundamenta. Al establecer el foco del desarrollo creativo en este aspecto procesual, se cuestionan conceptos de autoría, virtuosismo, obra e instrumento. La improvisación libre, o la creación desde la nada ante el público de material visual o sonoro, cuestiona el modelo de autoridad y autoría, presentándose como herramienta política en busca de un nuevo contexto creativo y artístico más apropiado en el entorno postdigital. Las aspiraciones de todo posicionamiento que defiende la improvisación como núcleo del proceso creativo se ven truncadas por el riesgo y la incertidumbre que suponen la exposición al público en tiempo real. El artista se refugia en elementos y recursos que sabe que funcionan por experiencias previas, y ese proceso ideal de libertad total se va consolidando poco a poco en un desarrollo controlado dentro de parámetros con garantías de resultados satisfactorios. El conflicto entre una creación en directo desde cero y la necesidad de estructuras con resultados garantizados deviene en territorio fértil para la creación, rico en tensiones y contradicciones. Este tipo de situaciones, dentro del conflicto, son propicias para la búsqueda de soluciones no conocidas y por tanto especialmente ricas para la innovación y la experimentación. 


\section{BIBLIOGRAFÍA}

Algorave Website, https://algorave.com/ (consultada el 11 de noviembre de 2019).

Andersson, Gustav, «The blank canvas paralysis», The modern nomad. 2012, https://www.themodernnomad.com/blank-canvas-paralysis/ (consultada el 11 de noviembre de 2019).

Atrinson, Sarah, y Helen W. Kennedy, 2018. Live cinema: cultures, economies, aesthetics. Londres: Ed. Bloomsbury Academic.

Bailey, Derek, «Free improvisation». Cortical Foundation. https://web.archive.org/web/20080605 044808/http://www.cortical.org/dbfree.html (consultada el 11 de noviembre de 2019).

Brown, Andrew R. «Code Jamming», M/C Journal 9.6. 2006, http://journal.media-culture.org. au/0612/03-brown.php (consultada el 11 de noviembre de 2019).

Brown, Andrew, y Andrew Sorensen. «Interacting with Generative Music through Live Coding». Contemporary Music Review 28 (2009).

Collins, Nick, Alex Mclean, Julian Rohrhuber, and Adrian Ward. «Live Coding in Laptop Performance», Organised Sound 8, no. 3 (2003).

Burland, K. y McLean, A. "Understanding live coding events", International Journal of Performance, vol. 12 (2016): 139-151.

FLAŠAR, Martin, «Listening with the Eyes: Remarks on Live Coding Performance» 2016. Media Archive performance. http://www.perfomap.de/map7/media-performance-on-gestures/ listening-with-the-eyes-remarks-on-live-coding-performance (consultada el 11 de noviembre de 2019).

Fortune, Stephen. "What on earth is livecoding?», Dazed. 2013, http://www.dazeddigital.com/ artsandculture/article/16150/1/what-on-earth-is-livecoding (consultada el 11 de noviembre de 2019).

Future Music Ed. "A brief history of Ableton Live», Music Radar. 2011, https://www.musicradar.com/tuition/tech/a-brief-history-of-ableton-live-357837 (consultada el 11 de noviembre de 2019).

Gree, Colin. The Free Jazz Collective. «Into the Maelstrom: Music, Improvisation and the Dream of Freedom: Before 1970 - David Toop (Bloomsbury Academic, 2016) ***1/2». 2017, http:// www.freejazzblog.org/2017/06/into-maelstrom-music-improvisation-and.html (consultada el 11 de noviembre de 2019).

KIrn, Peter. "Live coding group toplap celebrates days of live streaming, events», Create Digital Music. 2019. http://cdm.link/2019/02/live-coding-group-toplap-celebrates-days-of-livestreaming-events/ (consultada el 11 de noviembre de 2019).

Magnusson, Thor. «What is live coding?», Live Code Research Network. 2008. https://web.archive. org/web/20180626061052/http://www.livecodenetwork.org/what-is-live-coding/ (consultada el 11 de noviembre de 2019).

Magnusson, Thor and McLean, Alex. 2018. Performing with Patterns of Time. Oxford: Oxford University Press.

Mclean, Alex, Davide della Casa, Shelly Knotts, Sam Aaron, Andrew Brown and Juan A, Romero. «Toplab ManifestoDraft», Toplab. 2010, https://toplap.org/wiki/ManifestoDraft (consultada el 11 de noviembre de 2019). 
MI, Andrea. «Rave your algorithm. Interview with renick bell», Digicult. 2018, http://digicult.it/ articles/rave-your-algorithm-interview-with-renick-bell/ (consultada el $\overline{11 \text { de noviembre }}$ de 2019).

Munárriz Ortiz, Jaime. «Live Cinema: redefiniendo la narración audiovisual», Arte y Políticas de Identidad, vol. 9 (2013): 149-161.

NoTV. Lupercyclopedia. Petergreenaway. info. 2005, https://web.archive.org/web/20100529060345/ http://www.petergreenaway.info/ (consultada el 11 de noviembre de 2019).

Noys, Benjamin, «Tulse Luper Database: Peter Greenaway, the New Media Object and the Art of Exhaustion", Image and Narrative Online Magazine of the Visual Narrative. (2005) http:// www.imageandnarrative.be/inarchive/tulseluper/noys.htm (consultada el 11 de noviembre de 2019).

OhlenschläGer, Karin y Rico, Luis. «Interactivos?», Medialab Madrid. 2006, http://www.medialabmadrid.org/medialab/interactivos.php (consultada el 11 de noviembre de 2019).

Saladin, Matthieu. 2015. Points of Resistance and Criticism in Free Improvisation. Noise \& Capitalism. Guipúzcoa: Arteleku.

Surges, Greg. «Pure Data Live Coding 1-Multi-op F». 2012, https://www.youtube.com/watch?v=n LQMpUQ6It8 (consultada el 11 de noviembre de 2019).

Toop, David. 2016. Into the Maelstrom: Music, Improvisation and the Dream of Freedom: Before 1970. Londres: Bloomsbury Publishing. 
